\title{
Multiple gradations coupling effects in the bending of thin elastic FGM plates
}

\author{
V. Sladek, L. Sator \& J. Sladek \\ Institute of Construction and Architecture, \\ Slovak Academy of Sciences, Slovakia
}

\begin{abstract}
In this paper we present the derivation of the governing equations and boundary conditions for thin elastic plates with functionally graded Young's modulus and thickness of the plate. The derivation is based on the assumptions of the KirchhoffLove theory for bending of thin elastic plates. The combination of the transversal gradation of Young's modulus with in-plane gradations of Young's modulus and/or thickness of the plate yields the multiple gradations coupling effects. The main manifestation of these effects is a finite deflection of the plate even if it is subjected only to in-plane loading on the boundary edge. Of course the response of the plate is affected by coupling between the in-plane deformation and bending modes. In numerical simulations of the multiple gradations coupling effects, the meshless strong formulation with MLS (Moving Least Squares) approximation of field variables are employed for solution of formulated boundary value problems. Several numerical results are presented for illustration of the multiple gradations coupling effects in bending of thin elastic FGM (Functionally Graded Material) plates.

Keywords: Kirchhoff-Love theory, functional gradations of Young's modulus, variable thickness, transversal and in-plane gradations, strong formulation, MLS approximations.
\end{abstract}

\section{Introduction}

Plates are initially flat structural elements whose thickness is significantly smaller than the other dimensions. Because of small aspect ratio of thickness to in-plane dimensions, we can make certain assumptions on the deformation of plates. Having known the dependence of the stress-deformation state on the transversal 
coordinate, one can develop semi-integral formulation for bending of elastic plates by performing the integration across the thickness of the plate. Thus, the original $3 \mathrm{D}$ problem is recast into a $2 \mathrm{D}$ problem formulated for integral quantities with utilizing the integral characteristic such the bending stiffness. As long as the material is homogeneous, the bending and in-plane deformation modes are uncoupled and the boundary value problem for each of them can be solved separately.

It is known that functional gradation of material coefficients [1] can improve certain properties of structural elements. Thus, for instance, the bending stiffness of the plate is affected by the functional gradation of Young's modulus across the plate thickness. The bending stiffness is influenced also by variable thickness of the plate. But the change of the bending stiffness is not the only modification required to obtain correct behaviour of the FGM (Functionally Graded Material) plate as compared with the homogeneous one. The transversal gradation of Young's modulus yields coupling between the bending and in-plane deformation modes $[2,3]$. Therefore it is insufficient to change only the coefficients in the governing equations corresponding to homogeneous plate, but it is necessary to modify also the set of governing equations, in order to get the response of the FGM plate to applied loading. In this paper, we concise to the Kirchhoff-Love theory of bending of thin elastic plates (KLT). The correctly derived governing equations for FGM plates with variable plate thickness reveal also the other coupling effects, so called multiple gradations coupling effects, arising in the FGM plate with transversal gradation of Young's modulus and an additional in-plane gradation of at least one among the Young modulus and the plate thickness. In this paper, there are specified also the necessary conditions when an in-plane loading on the boundary edge of the plate results into finite deflections of the plate. Note that the governing equations involve several coupling terms which are proportional to various orders of derivatives of the field variables, Young's modulus and the plate thickness. Therefore it is difficult to assess even the order of the response to external loading and a numerical analysis is required. For the numerical solution of boundary value problems formulated in this paper, we propose to use the meshless strong formulation with MLS-approximation of field variables. Finally, we present several numerical results for illustration of the response of the FGM plate with variable thickness to external in-plane tension load on the plate boundary edge.

\section{Governing equations: derivation of coupling effects}

Let us consider a straight plate structure occupying the 3D domain

$$
\begin{aligned}
V=\left\{\forall\left(x_{1}, x_{2}, x_{3}\right) \in \mathbb{R}^{3} ; \mathbf{x}=\left(x_{1}, x_{2}\right) \in \Omega, x_{3} \in[-h / 2, h / 2]\right\} \\
=\Omega \times[-h / 2, h / 2] .
\end{aligned}
$$

In the KLT, we assume the linear expansion of the in-plane displacements with respect to the transversal coordinate $x_{3}$ and the transversal displacement $w(\mathbf{x})$ is assumed to be independent on $x_{3}$, i.e.: 


$$
v_{i}\left(\mathbf{x}, x_{3}\right)=\delta_{i \alpha}\left[u_{\alpha}(\mathbf{x})+x_{3} v_{\alpha, 3}(\mathbf{x}, 0)\right]+\delta_{i 3} w(\mathbf{x}), \alpha \in\{1,2\},
$$

with $u_{\alpha}(\mathbf{x})$ being the in-plane displacements in the mid-plane.

It can be seen that the shear strains are independent on the transversal coordinate

$$
e_{\alpha 3}\left(\mathbf{x}, x_{3}\right)=\left(v_{\alpha, 3}\left(\mathbf{x}, x_{3}\right)+v_{3, \alpha}\left(\mathbf{x}, x_{3}\right)\right) / 2=\left(v_{\alpha, 3}(\mathbf{x}, 0)+w_{, \alpha}(\mathbf{x})\right) / 2 .
$$

Then, the requirement of vanishing shear stresses on the top and bottom of the plate yields

$$
v_{\alpha, 3}(\mathbf{x}, 0)=-w_{, \alpha}(\mathbf{x}) .
$$

Hence and from (1), we obtain

$$
v_{i}\left(\mathbf{x}, x_{3}\right)=\delta_{i \alpha}\left[u_{\alpha}(\mathbf{x})-x_{3} w_{, 3}(\mathbf{x})\right]+\delta_{i 3} w(\mathbf{x})
$$

and the strains are given by

$$
\begin{gathered}
e_{\alpha \beta}\left(\mathbf{x}, x_{3}\right)=\varepsilon_{\alpha \beta}(\mathbf{x})-x_{3} w_{, \alpha \beta}(\mathbf{x}), \varepsilon_{\alpha \beta}(\mathbf{x}):=\left(u_{\alpha, \beta}(\mathbf{x})+u_{\alpha, \beta}(\mathbf{x})\right) / 2, \\
\varepsilon_{\alpha 3}(\mathbf{x})=\varepsilon_{33}(\mathbf{x})=0, e_{k k}\left(\mathbf{x}, x_{3}\right)=u_{\gamma, \gamma}(\mathbf{x})-x_{3} w_{, \gamma \gamma}(\mathbf{x}) .
\end{gathered}
$$

Thus, the shear strains are vanishing not only on the top and bottom of the plate, but everywhere in the plate. This is the main handicap of the KLT. Nevertheless, the KLT works well in case of thin elastic plates.

According to the Hooke's law, the stress tensor components can be written as

$$
\begin{gathered}
\sigma_{\alpha \beta}\left(\mathbf{x}, x_{3}\right)=\frac{E}{1-v^{2}} \frac{1-v}{H}\left\{\tau_{\alpha \beta}^{(u)}(\mathbf{x})-x_{3} \tau_{\alpha \beta}^{(w)}(\mathbf{x})\right\}, \sigma_{\alpha 3}\left(\mathbf{x}, x_{3}\right)=0, \\
\sigma_{33}\left(\mathbf{x}, x_{3}\right)=\frac{E}{1-v^{2}} \frac{1-v}{H}\left\{u_{\gamma, \gamma}(\mathbf{x})-x_{3} w_{, \gamma \gamma}(\mathbf{x})\right\},
\end{gathered}
$$

with $\tau_{\alpha \beta}^{(u)}(\mathbf{x}):=H \varepsilon_{\alpha \beta}(\mathbf{x})+v \delta_{\alpha \beta} u_{\gamma, \gamma}(\mathbf{x}), \tau_{\alpha \beta}^{(w)}(\mathbf{x}):=H w_{, \alpha \beta}(\mathbf{x})+v \delta_{\alpha \beta} w_{, \gamma \gamma}(\mathbf{x})$, $H=1-v$ for plane stress problems, while $H=1-2 v$ otherwise.

Thus, having known the transversal dependence, the plate bending problem can be analysed as a $2 \mathrm{D}$ problem. The governing equations for the field variables $u_{\alpha}(\mathbf{x})$ and $w(\mathbf{x})$ can be derived in the semi-integral formulation from the variational principle

$$
\left.\int_{\Omega}^{h / 2} \int_{-h / 2}^{h} \sigma_{i j} \delta e_{i j} d x_{3}\right) d \Omega-\int_{\Omega} q \delta w d \Omega-h \int_{\partial \Omega} \bar{t}_{\alpha} \delta u_{\alpha} d \Gamma=0,
$$

where $q$ is surface density of the transversal load and $\bar{t}_{\alpha}(\mathbf{x})$ is the in-plane load applied on the boundary edge of the plate. Performing the integration across the plate thickness explicitly, we receive

$$
\begin{gathered}
-\int_{\Omega}\left\{T_{\alpha \beta, \beta} \delta u_{\alpha}+\left(M_{\alpha \beta, \alpha \beta}+q\right) \delta w\right\} d \Omega+ \\
+\int_{\partial \Omega}\left(\left(n_{\beta} T_{\alpha \beta}-h \bar{t}_{\alpha}\right) \delta u_{\alpha}-n_{\alpha} n_{\beta} M_{\alpha \beta} \delta\left(\frac{\partial w}{\partial \mathbf{n}}\right)+V \delta w\right) d \Gamma-\delta w \sum \llbracket T \rrbracket=0,
\end{gathered}
$$


where

$$
\begin{gathered}
T_{\alpha \beta}(\mathbf{x}):=\int_{-h / 2}^{h / 2} \sigma_{\alpha \beta}\left(\mathbf{x}, x_{3}\right) d x_{3}, M_{\alpha \beta}(\mathbf{x}):=\int_{-h / 2}^{h / 2} x_{3} \sigma_{\alpha \beta}\left(\mathbf{x}, x_{3}\right) d x_{3}, \\
\tilde{T}:=t_{\alpha} n_{\beta} M_{\alpha \beta}, V:=n_{\alpha} M_{\alpha \beta, \beta}+\frac{\partial T}{\partial \mathbf{t}}, \llbracket T \rrbracket:=T\left(\mathbf{x}^{c}+0\right)-T\left(\mathbf{x}^{c}-0\right) .
\end{gathered}
$$

In view of (8), the governing equations in the semi-integral formulation are given as

$$
\begin{gathered}
T_{\alpha \beta, \beta}=0, \\
M_{\alpha \beta, \alpha \beta}=-q,
\end{gathered}
$$

and the boundary conditions should obey the following restriction equations at each boundary point

$$
\begin{gathered}
\left.\left(n_{\beta} T_{\alpha \beta}-h \bar{t}_{\alpha}\right) \delta u_{\alpha}\right|_{\partial \Omega}=0 \\
\left.n_{\alpha} n_{\beta} M_{\alpha \beta} \delta\left(\frac{\partial w}{\partial \mathbf{n}}\right)\right|_{\partial \Omega}=0,\left.\left(V-\delta\left(\mathbf{x}-\mathbf{x}^{c}\right) \sum_{c} \llbracket T \rrbracket\right) \delta w\right|_{\partial \Omega}=0 .
\end{gathered}
$$

It is appropriate to use dimensionless formulation specified as follows

$$
\begin{gathered}
x_{\alpha}^{*}=\frac{x_{\alpha}}{L}, x_{3}^{*}=\frac{x_{3}}{h_{0}}, u_{\alpha}^{*}=\frac{u_{\alpha}}{h_{0}}, w^{*}=\frac{w}{h_{0}}, \frac{\partial}{\partial x_{\alpha}}=\frac{1}{L} \frac{\partial}{\partial x_{\alpha}^{*}}, \\
D_{0}:=\frac{E_{0} h_{0}^{3}}{12\left(1-v^{2}\right)}, q^{*}:=\frac{L^{4}}{D_{0} h_{0}} q .
\end{gathered}
$$

Let us consider a plate with continuously variable thickness $h(\mathbf{x})=h_{0} h^{*}(\mathbf{x})$ and the Young modulus

$$
E\left(\mathbf{x}, x_{3}\right)=E_{0} E_{H}^{*}(\mathbf{x}) E_{V}^{*}(z),
$$

with assuming the power-law gradation over the thickness of the plate

$$
E_{V}^{*}(z)=1+\zeta\left(\frac{1}{2}+z\right)^{p}, z:=\frac{x_{3}}{h} .
$$

Then, the integrations with respect to $x_{3}$ in (9) can be expressed in terms of integrals

$$
\int_{-1 / 2}^{1 / 2} z^{k} E_{V}^{*}(z) d z=d_{(0) k}+\zeta d_{(p) k}
$$

which are given in closed form as

$$
d_{(0) k}:=\int_{-1 / 2}^{1 / 2} z^{k} d z=\frac{1+(-1)^{k}}{(k+1) 2^{k+1}},
$$




$$
\begin{gathered}
d_{(p) k}:=\int_{-1 / 2}^{1 / 2} z^{k}\left(\frac{1}{2} \pm z\right)^{p} d z=( \pm 1)^{k} \int_{0}^{1}\left(y-\frac{1}{2}\right)^{k} y^{p} d y= \\
=( \pm 1)^{k} \sum_{j=0}^{k}\left(\begin{array}{c}
k \\
j
\end{array}\right)\left(-\frac{1}{2}\right)^{j} \frac{1}{p+1+k-j} .
\end{gathered}
$$

Now, we may write

$$
\begin{gathered}
T_{\alpha \beta}^{*}(\mathbf{x})=\frac{L^{2}}{D_{0}} T_{\alpha \beta}(\mathbf{x})=C^{(u u)}(\mathbf{x}) \tau_{\alpha \beta}^{*(u)}(\mathbf{x})+C^{(u w)}(\mathbf{x}) \tau_{\alpha \beta}^{*(w)}(\mathbf{x}) \\
M_{\alpha \beta}^{*}=\frac{L^{2}}{D_{0} h_{0}} M_{\alpha \beta}=C^{(w u)}(\mathbf{x}) \tau_{\alpha \beta}^{*(u)}(\mathbf{x})+C^{(w w)}(\mathbf{x}) \tau_{\alpha \beta}^{*(w)}(\mathbf{x})
\end{gathered}
$$

where the superscript $\left(^{*}\right)$ is omitted in Cartesian coordinates and we have used the notations

$$
\begin{gathered}
\tau_{\alpha \beta}^{*(u)}:=\frac{H}{2}\left(u_{\alpha, \beta}^{*}(\mathbf{x})+u_{\beta, \alpha}^{*}(\mathbf{x})\right)+v \delta_{\alpha \beta} u_{\gamma, \gamma}^{*}(\mathbf{x})=\frac{L}{h_{0}} \tau_{\alpha \beta}^{(u)}, \\
\tau_{\alpha \beta}^{*(w)}:=H w_{, \alpha \beta}^{*}(\mathbf{x})+v \delta_{\alpha \beta} \nabla^{2} w^{*}(\mathbf{x})=\frac{L^{2}}{h_{0}} \tau_{\alpha \beta}^{(w)} \\
C^{(u u)}(\mathbf{x}):=12 \frac{1-v}{H} \frac{L}{h_{0}}\left(1+\zeta d_{(p) 0}\right) D_{1 H}^{*}(\mathbf{x}), \\
C^{(u w)}(\mathbf{x}):=-12 \frac{1-v}{H} \zeta d_{(p) 1} D_{2 H}^{*}(\mathbf{x})=-\frac{h_{0}}{L} C^{(w u)}(\mathbf{x}), \\
C^{(w w)}(\mathbf{x}):=-12 \frac{1-v}{H}\left[d_{(0) 2}+\zeta d_{(p) 2}\right] D_{3 H}^{*}(\mathbf{x}), \\
D_{j H}^{*}(\mathbf{x}):=\left(h^{*}(\mathbf{x})\right)^{j} E_{H}^{*}(\mathbf{x}) .
\end{gathered}
$$

Now, in view of equations (17)-(19), the governing equations become

$$
\begin{gathered}
\frac{L}{h_{0}}\left(1+\zeta d_{(p) 0}\right)\left\{D_{1 H, \beta}^{*}\left[\frac{H}{2}\left(u_{\alpha, \beta}^{*}+u_{\beta, \alpha}^{*}\right)+v \delta_{\alpha \beta} u_{\gamma, \gamma}^{*}\right]+\right. \\
\left.+D_{1 H}^{*} \frac{1}{2}\left[H s_{\alpha}^{*}+(H+2 v) u_{\gamma, \gamma \alpha}^{*}\right]\right\}- \\
-\zeta d_{(p) 1}\left\{D_{2 H, \beta}^{*}\left[H w_{, \alpha \beta}^{*}+v \delta_{\alpha \beta} m^{*}\right]+D_{2 H}^{*}(H+v) m_{, \alpha}^{*}\right\}=0, \\
\frac{L}{h_{0}} \zeta d_{(p) 1}\left\{D_{2 H, \alpha \beta}^{*}\left[H u_{\alpha, \beta}^{*}+v \delta_{\alpha \beta} u_{\gamma, \gamma}^{*}\right]+\right. \\
\left.+D_{2 H, \beta}^{*}\left[H s_{\beta}^{*}+(H+2 v) u_{\gamma, \gamma \beta}^{*}\right]+D_{2 H}^{*}(H+v) s_{\alpha, \alpha}^{*}\right\}-
\end{gathered}
$$




$$
\begin{aligned}
&-\left[d_{(0) 2}+\zeta d_{(p) 2}\right]\left\{D_{3 H, \alpha \beta}^{*}\left[H w_{, \alpha \beta}^{*}+v \delta_{\alpha \beta} m^{*}\right]+2 D_{3 H, \beta}^{*}(H+v) m_{, \beta}^{*}+\right. \\
&\left.+D_{3 H}^{*}(H+v) m_{, \beta \beta}^{*}\right\}=-\frac{H}{12(1-v)} q^{*} \\
& \nabla^{2} w^{*}-m^{*}=0 \\
& \nabla^{2} u_{\alpha}^{*}-s_{\alpha}^{*}=0
\end{aligned}
$$

where the introduction of new field variables $m^{*}$ and $s_{\alpha}^{*}$ by equations (22) and (23) decreases the order of derivatives by decomposing the original PDE into the set of the 2 nd order PDE given by equations (20)-(23). Such decomposition is necessary in order to avoid inaccuracy of numerical evaluation of higher than 2 nd order derivatives of field variables.

If the Young modulus is not graded across the plate thickness $(\zeta=0)$, the system of the governing equations (20)-(23) is splitting into two subsystems which can be solved separately for in-plane displacements and the deflection. Furthermore, if $\zeta \neq 0$, the finite deflections are a source for in-plane displacements even if $D_{2 H}^{*}=$ const, i.e. if both the plate thickness and Young's modulus factor $E_{H}^{*}(\mathbf{x})$ are constant in $\Omega$. On the other hand, finite values of inplane displacements can be insufficient for creation of deflections in FGM plate with transversal gradation of Young's modulus and without transversal loading. In such a case, finite deflections can be created if at least one of the following conditions is satisfied: (i) transversal gradation of Young's modulus $\zeta \neq 0 \wedge$ strongly non-linear distribution of in-plane displacements $\left(\nabla^{2} u_{\alpha}^{*}\right)_{, \alpha} \neq 0$; (ii) transversal gradation of Young's modulus $\zeta \neq 0 \wedge$ in-plane gradation $D_{2 H, \beta}^{*} \neq 0 \wedge$ non-linear distribution of in-plane displacements $u_{\alpha, \beta \gamma}^{*} \neq 0$; (iii) transversal gradation of Young's modulus $\zeta \neq 0 \wedge$ in-plane gradation $D_{2 H, \alpha \beta}^{*} \neq 0 \wedge$ linear distribution of in-plane displacements $u_{\alpha, \beta}^{*} \neq 0$. Bearing in mind the expressions

$$
\begin{gathered}
D_{j H, \beta}^{*}:=j\left(h^{*}\right)^{j-1} h_{, \beta}^{*} E_{H}^{*}+\left(h^{*}\right)^{j} E_{H, \beta}^{*} \\
D_{j H, \alpha \beta}^{*}:=\left[j(j-1)\left(h^{*}\right)^{j-2} h_{, \alpha}^{*} h_{, \beta}^{*}+j\left(h^{*}\right)^{j-1} h_{, \alpha \beta}^{*}\right] E_{H}^{*}+ \\
+j\left(h^{*}\right)^{j-1}\left[h_{, \beta}^{*} E_{H, \alpha}^{*}+h_{, \alpha}^{*} E_{H, \beta}^{*}\right]+\left(h^{*}\right)^{j} E_{H, \alpha \beta}^{*},
\end{gathered}
$$

we can see that at least one of the quantities $\left\{h^{*}(\mathbf{x}), E_{H}^{*}(\mathbf{x})\right\}$ is to be graded in $\Omega$ additionally to the transversal gradation of the Young modulus in order to satisfy the necessary conditions (ii) and/or (iii) for creation of finite deflection response to in-plane deformations of the FGM plate. Then, we are talking about the multiple gradations coupling effects. 
The set of the governing equations (20)-(23) should be supplemented by boundary condition on the plate edge. For in-plane deformations, we have two kinds of boundary conditions: (i) essential conditions: $u_{\alpha}(\mathbf{x})=\bar{u}_{\alpha}(\mathbf{x})$ on $\partial \Omega_{u}$; (ii) natural conditions: $n_{\beta} T_{\alpha \beta}=h \bar{t}_{\alpha}$ on $\partial \Omega_{t}$, which can be combined with the three kinds of the "plate bending" boundary conditions: (a) clamped edge: $w(\mathbf{x})=0,(\partial w / \partial n)(\mathbf{x})=0 ; \quad$ (b) simply supported edge: $w(\mathbf{x})=0$, $n_{\alpha} n_{\beta} M_{\alpha \beta}(\mathbf{x})=0$; (c) free edge: $n_{\alpha} n_{\beta} M_{\alpha \beta}(\mathbf{x})=0, V(\mathbf{x})-\delta\left(\mathbf{x}-\mathbf{x}^{c}\right) \sum_{c} \llbracket T \rrbracket=0$.

\section{Numerical implementation}

For numerical solution of boundary value problems in this paper, we employ the strong formulation with meshless approximation of field variables by Moving Least Square (MLS) approximation [4]. The nodal points are freely distributed in the analysed domain and on its boundary without creating any connectivity among the nodes. The approximations of in-plane displacements as well as deflections at a point are expressed in terms of nodal unknowns and shape functions corresponding to certain nodes selected according to chosen weight functions. In the standard differentiation approach, the derivatives of field variables are approximated in terms of the same nodal unknowns as primary fields and the derivatives of the shape functions [5]. The order of the derivatives can be reduced in the modified differentiation approach [6], when even the derivatives of filed variables are approximated by using the shape functions and the nodal unknowns are expressed in terms of the 1 st order derivatives of the shape functions at nodal points. For more details, we refer the reader to the works $[5,6]$.

\section{Numerical examples}

In order to illustrate the multiple gradations coupling effects, we shall consider the FGM square plate (Figure 1) with the transversal power-law gradation of Young's modulus by (14) and possible in-plane power-law gradations of the plate thickness and the Young modulus factor $E_{H}^{*}(\mathbf{x})$.

$$
\begin{gathered}
E_{H}^{*}(\mathbf{x})=1+\varepsilon\left(x_{1}\right)^{\alpha}, E_{H}^{*}(\mathbf{x})=1+\varepsilon\left(1-x_{1}\right)^{\alpha} \\
h^{*}(\mathbf{x})=1+\kappa\left(x_{1}\right)^{s}, h^{*}(\mathbf{x})=1+\kappa\left(x_{1}\right)^{s}
\end{gathered}
$$

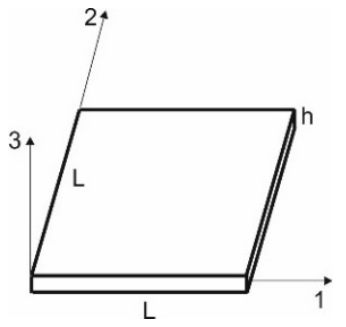

Figure 1: Sketch of the plate. 
The following boundary conditions are assumed:

- clamped boundary edge (for the bending mode)

$$
\left.w^{*}\right|_{\partial \Omega}=0,\left.\frac{\partial w^{*}}{\partial \mathbf{n}}\right|_{\partial \Omega}=0,\left.q^{*}\right|_{\Omega}=0
$$

- uniform tension $\overline{t_{i}}=\delta_{i 1}$ is applied on the edge $x_{1}^{*}=1 ; u_{1}^{*}=0$ on the edge $x_{1}^{*}=0$, while the rest of the boundary $\partial \Omega$ is traction free (for in-plane deformation mode), i.e.

$$
\begin{gathered}
\frac{L}{h_{0}}\left(1+\zeta d_{(p) 0}\right)\left\{D_{1 H}^{*}\left[\frac{H}{2}\left(u_{\alpha, 1}^{*}+u_{1, \alpha}^{*}\right)+v \delta_{\alpha 1} u_{\gamma, \gamma}^{*}\right]+\right. \\
\left.-\zeta d_{(p) 1} D_{2 H}^{*}(H+v) \delta_{\alpha 1} m^{*}\right\}\left.\right|_{x_{1}^{*}=1}=\left.\delta_{\alpha 1} \frac{H h_{0} h^{*}}{12(1-v)}\right|_{x_{1}^{*}=1} \overline{t_{1}}, \\
\left.\frac{L}{h_{0}}\left(1+\zeta d_{(p) 0}\right) D_{1 H}^{*} \frac{H}{2}\left(u_{2,1}^{*}+u_{1,2}^{*}\right)\right|_{x_{1}^{*}=0}=0,\left.u_{1}^{*}\right|_{x_{1}^{*}=0}=0, \\
\frac{L}{h_{0}}\left(1+\zeta d_{(p) 0}\right)\left\{D_{1 H}^{*}\left[\frac{H}{2}\left(u_{\alpha, 2}^{*}+u_{2, \alpha}^{*}\right)+v \delta_{\alpha 2} u_{\gamma, \gamma}^{*}\right]+\right. \\
\left.-\zeta d_{(p) 1} D_{2 H}^{*}(H+v) \delta_{\alpha 2} m^{*}\right\}\left.\right|_{x_{2}^{*}=1 \text { or } 0}=0,
\end{gathered}
$$

where we used certain simplifications on the clamped edges $x_{1}^{*}=1$ or $x_{1}^{*}=0$ :

$$
\begin{gathered}
w^{*}=0=\text { const and } w_{, 1}^{*}=0=\text { const } \Rightarrow w_{, 2}^{*}=0, w_{, 22}^{*}=0, w_{, 12}^{*}=0, \\
m^{*}=w_{, 11}^{*},
\end{gathered}
$$

while on the clamped edges $x_{2}^{*}=1$ or $x_{2}^{*}=0$ :

$$
\begin{gathered}
w^{*}=0=\text { const and } w_{, 2}^{*}=0=\text { const } \Rightarrow w_{, 1}^{*}=0, w_{, 11}^{*}=0, w_{, 21}^{*}=0, \\
m^{*}=w_{, 22}{ }^{*} .
\end{gathered}
$$

In numerical calculations, we have utilized uniform distribution of $26 \times 26$ nodes (with $\delta$ being the distance between two neighbour nodes), cubic polynomial basis, Gaussian weights with the radius of support domain $3.001 \times \delta$ and shape parameter $c=\delta$.

When we considered only the transversal gradation of the Young modulus (YM), the in-plane displacements were influenced by the level of gradation (see Figure 2), but there was no response in deflections $\left(w^{*} \sim 10^{-11}\right)$. 


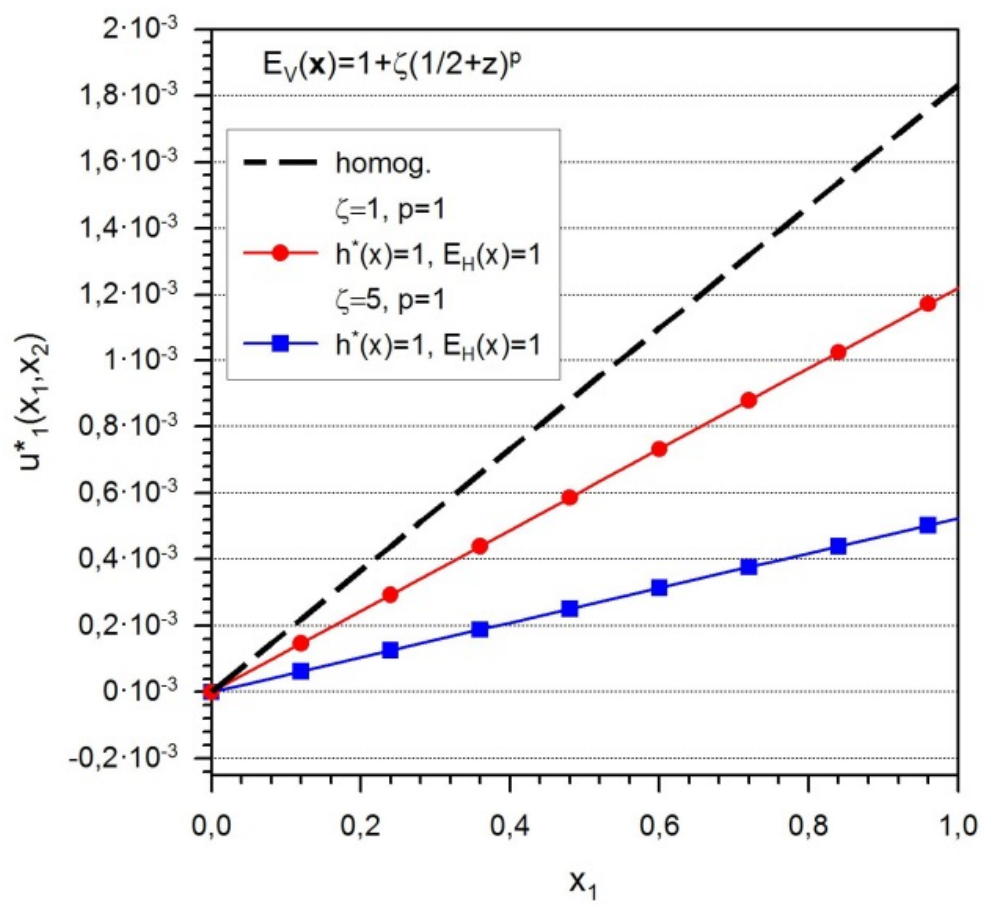

Figure 2: The response in homogeneous and two FGM plates with transversal gradations of YM.

If the transversal gradation of YM is combined with variable thickness of the plate and/or in-plane gradation of YM, the multiple gradations coupling effects take place and finite deflections are created even by in-plane loading. The response of in-plane displacements become non-linear (Figure 3). In this case, the in-plane gradation of Young's modulus is increasing from the fixed edge toward the loaded edge and the plate thickness is constant.

Finally, we present the response in the FGM plates with combined transversal gradation of YM and in-plane gradations of the plate thickness increasing from the fixed edge toward the loaded one. It can be seen from Figures 3 and 4 that the coupling is more expressive in the latter case, though the responses for $u_{1}$ displacements are very similar in both cases.

\section{Conclusions}

The multiple gradations coupling effects are described within the Kirchhoff-Love theory for bending of thin elastic plates. These effects arise in the FGM plate with transversal gradation of Young's modulus and an additional in-plane gradation of at least one among the Young modulus and the plate thickness. The 2D formulation, i.e. the governing equations and boundary conditions, are derived for 

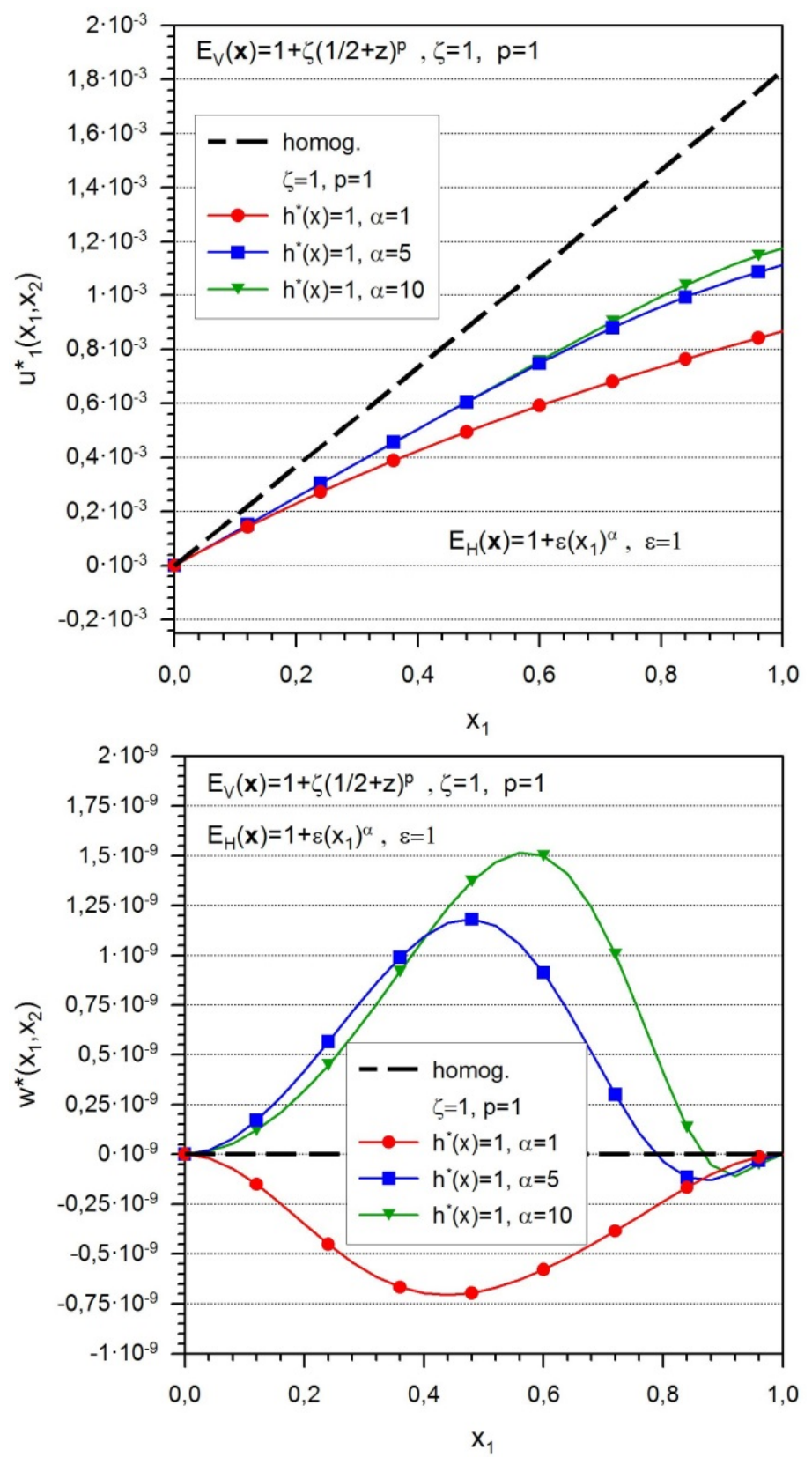

Figure 3: The response in homogeneous and FGM plates with combined transversal gradation of $\mathrm{YM}$ and in-plane gradations of $\mathrm{YM}$ increasing from the fixed edge toward the loaded edge. The results are plotted along the line $\left(x_{1}, x_{2}=0.5\right)$. 

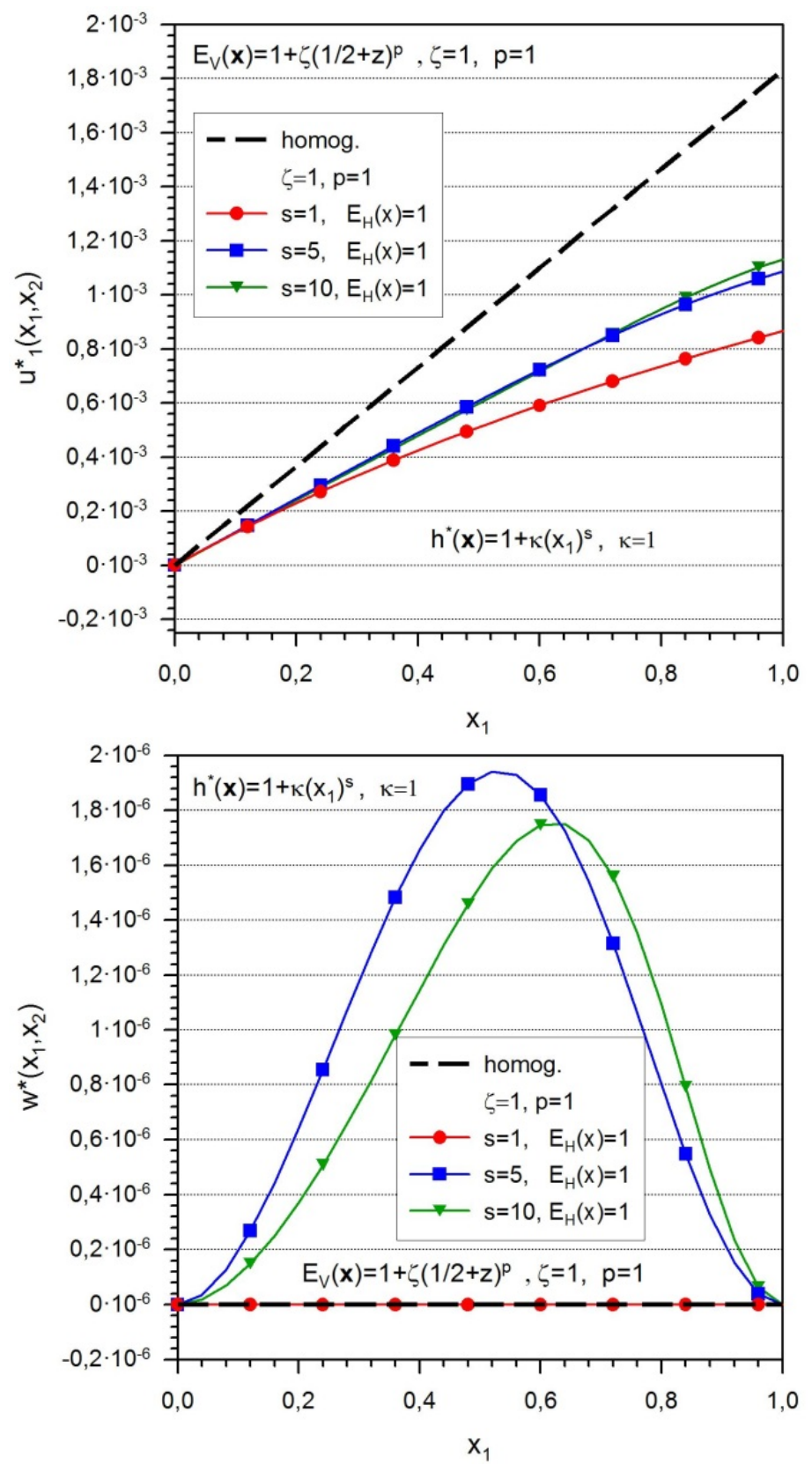

Figure 4: The response in homogeneous and FGM plates with combined transversal gradation of YM and in-plane gradations of YM or plate thickness increasing from the loaded edge toward the fixed edge. The results are plotted along the line $\left(x_{1}, x_{2}=0.5\right)$. 
coupled bending and in-plane deformation modes starting from the $3 \mathrm{D}$ variational formulation for FGM plates with variable thickness and with applying the Kirchhoff-Love assumptions. Analysing the derived governing PDE with variable coefficients, we have specified the necessary conditions for creation of finite deflections in FGM plates subjected to in-plane traction load on the boundary edge. The numerical simulations for some illustrative examples document creation of finite deflections of the plate as well as the influence of multiple gradations (including coupling between bending and in-plane deformation modes) on the inplane displacements.

\section{Acknowledgement}

This work was partially supported by the Slovak Research and Development Agency under the contract No. APVV-14-0440.

\section{References}

[1] Birman V., Byrd L.W., Modeling and analysis of functionally graded materials and structures. Appl. Mech. Rev. (ASME). 60, pp. 195-216, 2007.

[2] Sladek, V., Sladek, J., Sator, L., Physical decomposition of thin plate bending problems and their solution by mesh-free methods. Engineering Analysis with Boundary Elements. 37, pp. 348-365, 2013.

[3] Sator L., Sladek V., Sladek J., Coupling effects in elastic analysis of FGM composite plates by mesh-free methods. Composite Structures. 115, pp. 100$110,2014$.

[4] Lancaster, P., Salkauskas, K., Surfaces generated by moving least square method. Math. Comput. 37, pp. 141-158, 1981.

[5] Sladek, V., Sladek, J., Zhang, Ch., Computation of stresses in nonhomogeneous elastic solids by local integral equation method: a comparative study. Computational Mechanics. 41, pp. 827-845, 2008.

[6] Sladek, V., Sladek, J., Local integral equations implemented by MLSapproximation and analytical integrations. Engineering Analysis with Boundary Elements. 34, pp. 904-913, 2010. 